

\title{
EVALUACIÓN TÉRMICA Y LUMÍNICA EN PROTOTIPOS DE CUBIERTAS LIGERAS, PARA CLIMA CÁLIDO HÚMEDO
}

\section{THERMALAND LIGHTING EVALUATION IN LIGHT ROOF PROTOTYPES, FOR HOT HUMID CLIMATES}

\author{
EDISON BOLIVAR ORTIZ-ZAMBRANO \\ Ingeniero Civil \\ Estudiante de posgrado de la Maestría Académica \\ con Trayectoria Profesional en Ingeniería Civil: \\ Mención en Construcción de Vivienda Social, Instituto de Posgrado \\ Universidad Técnica de Manabí, Portoviejo, Ecuador \\ https://orcid.org/0000-0001-8858-1361 \\ bolivar_ortiz@livecom
}

JEFFERSONTORRES-QUEZADA

Doctor en Arquitectrura, Energía y Medio Ambiente

Docente-Investigador

Universidad Católica de Cuenca Cuenca, Ecuador

https://orcid.org/0000-0002-1832-1369

jefferson.torres@ucacue.edu.ec

\author{
JOSÉ FABIÁN VÉLIZ-PÁRRAGA \\ Doctor en Ciencias Técnicas \\ Docente Universidad Técnica de Manabi \\ Portoviejo, Ecuador \\ https://orcid.org/0000-0001-5356-4537 \\ jfveliz@hotmail.com
}

\section{RESUMEN}

El techo de chapa de zinc es uno de los elementos más populares en la arquitectura latinoamericana, y en muchas otras regiones con climas cálido húmedo. Crear alternativas lumínicas y térmicas enfocadas en esta tipología implicaría profundos beneficios en el campo ambiental y social. El presente estudio, realizado en Manabí-Ecuador, evalúa tres prototipos de cubiertas ligeras, combinando el material Zinc con el PVC, con el fin de determinar la correcta configuración del material translucido para crear ambientes que estén dentro de los parámetros térmicos y lumínicos. Los resultados indican que el modelo de soluciones empíricas presenta la menor variación de temperatura interior, con un 32,63\%, a diferencia de los 32,97\% del modelo tipo cruz y de los 34,40\% del modelo de franjas laterales. Adicionalmente, se evidenció que la mayor influencia de radiación solar sobre la cubierta se registra desde las 13:00 h hasta las 14:00 h aproximadamente.

\section{Palabras clave}

Ecuador, vivienda, techos, comportamiento térmico interior

\section{ABSTRACT}

The zinc sheet roof is one of the most popular elements in Latin American architecture, and in many other regions with warm humid climates. Creating lighting and thermal alternatives focused on this typology would imply major benefits in the environmental and social fields. This study carried out in Manabí, Ecuador, evaluates three prototypes of light roofs, combining zinc with PVC, in order to determine the correct configuration of translucent material to create environments that are within thermal and lighting parameters. The results indicate that the empirical solutions model has the lowest variation in indoor temperature, with $32.63 \%$, unlike the $32.97 \%$ of the cross-type model, and the $34.40 \%$ of the side strip model. Additionally, it was seen that the greatest influence of solar radiation on the roof is recorded from 1:00 p.m. to 2 p.m. approximately. 

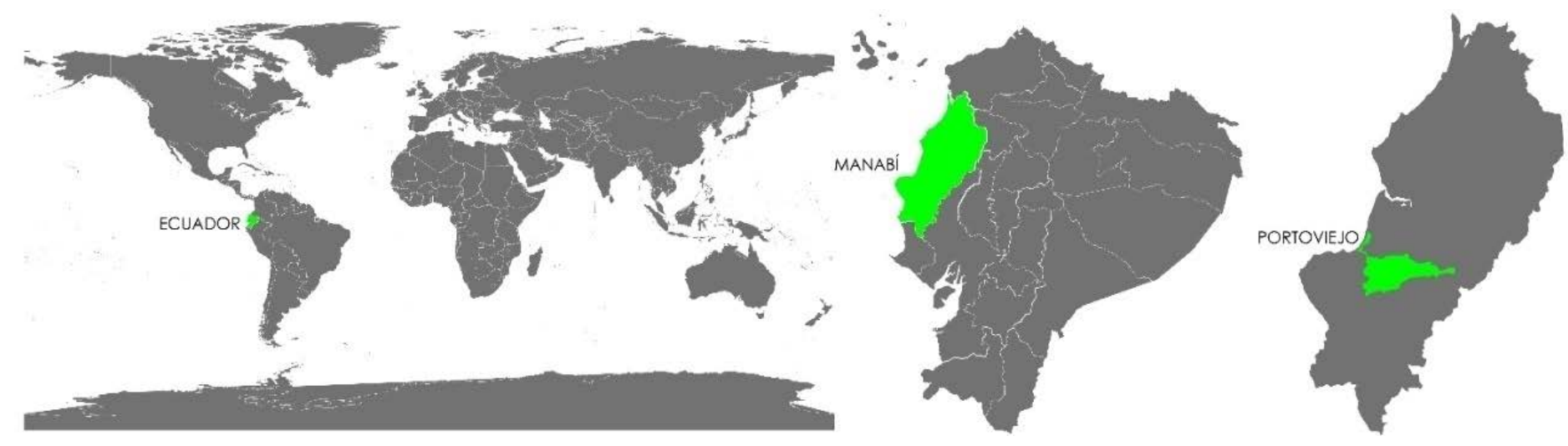

Figura 1. Ubicación geográfica de la zona de estudio. Fuente: Elaborado por los autores.

\section{INTRODUCCIÓN}

La cubierta es la parte superior de una edificación, cuya función principal es la de proteger su interior ante las inclemencias meteorológicas y amenazas externas. Este elemento estructural es clasificado por su forma, constitución material y complejidad (Hernández-Salomón, Rizo-Aguilera y Frómeta-Salas, 2017). Una de las múltiples opciones en el mercado ecuatoriano y la más recurrente en la construcción de vivienda de interés social (VIS) es la cubierta metálica, caracterizada por ser una lámina de acero ondulada recubierta por zinc y aluminio, conocido como Aluzinc (Véliz-Párraga y González-Couret, 2019). Esta tipología de cubierta resalta como una solución económica y resistente, de fácil aplicación y buen aspecto visual, no obstante, debido a la simple constitución estructural y a su baja transmitancia térmica, no presta las condiciones óptimas de aislamiento, lo que provoca un acelerado paso del flujo del calor en las edificaciones, generando ambientes inconfortables al interior (Rodríguez, 2017). En ciertas ocasiones se utiliza la teja translúcida de policloruro de vinilo (PVC), derivado de la sal (57\%) y del petróleo (43\%) (plasticseurope, 2021), sin embargo, la utilización de esta es reducida por presentar un alto costo, además de que una de sus características es permitir el paso de la luz cenital, que también produce un aumento en la intensidad calorífica al interior de la vivienda. La chapa de zinc, por su constitución química, tiene la cualidad de reflectancia, que la chapa de PVC no posee; esta propiedad de reflejar la luz es una de las más importantes, ya que indica la cantidad de energía radiante solar que una superficie refleja, por lo tanto, es determinante en el comportamiento térmico de los techados (Alchapar, Correa y Cantón, 2012). Asimismo, esta superficie está en alta dependencia de la posición del sol y, por ende, del espacio geográfico donde interactúa con el medio ambiente, lo cual se debe al ángulo de inclinación de los rayos solares que varían en latitud $\left(0^{\circ}\right.$ $90^{\circ}$ Norte y Sur y longitud $\left(0^{\circ}-180^{\circ}\right.$ Este y Oeste) del globo terráqueo (Oster, 2021, p. 39). Las regiones cercanas a la latitud $0^{\circ}$ tienen como condición que la cubierta recibe los rayos solares de manera perpendicular durante todo el año, como es el caso de Ecuador (Torres, Coch y Isalgué, 2019).

El mencionado país está conformado por 24 provincias. Limitando con el Océano Pacífico se encuentran la provincia Manabí y, dentro de ella, el Cantón Portoviejo (Figura 1). Esta provincia se distingue por tener temperaturas promedio anuales entre $21^{\circ} \mathrm{C}$ a $29^{\circ} \mathrm{C}$, divididas en dos periodos climáticos, el seco y el húmedo (Macías, 2018).

Según el Instituto Nacional de Meteorología e Hidrología [INAMHI], para el 29 de octubre del 2020, finales de la época seca, se registraron temperaturas máximas absolutas de $32^{\circ} \mathrm{C}$ (El Universo, 2020); para el 30 de marzo del 2018, las temperaturas máximas absolutas fueron de $34.9^{\circ} \mathrm{C}$ (El Diario, 2018) y, para el 10 de julio del 2014, final de la época húmeda, se alcanzaron temperaturas máximas absolutas de $35.5^{\circ} \mathrm{C}$ (INAMHI, 2014). Este corte longitudinal retrospectivo a través del tiempo indica el fenómeno climático que se produce a las cercanías de la latitud $0^{\circ}$. En ese contexto, debe considerarse que la radiación solar recibida por la cubierta produce más de un tercio de la ganancia térmica al interior de una vivienda (Osuna-Motta, Herrera-Cáceres y López-Bernal, 2017) y que las tipologías soluciones habitacionales del Cantón Portoviejo, donde prevalecen las edificaciones de baja altura (Couret y Párraga, 2019), cuyas cubiertas están elaboradas predominantemente por láminas de acero recubiertas por zinc y aluminio (Véliz-Párraga y GonzálezCouret, 2019). Queda de manifiesto, entonces, que es dicho elemento constructivo el principal responsable de los aportes de calor hacia el interior de las viviendas.

\section{ESTRATEGIAS PASIVAS PARA LOS TECHOS}

En referencia al desempeño térmico de los techos, se han estudiado diferentes aspectos y estrategias generales como ubicación y orientación; incremento de pérdidas por convección al aprovechar la dirección de los vientos; compacidad o factor de forma; superficie útil; y volumen total de la envolvente térmica y la masa térmica de sus componentes (Sisternes, 2019). 
Todas estas estrategias se han aplicado con el objetivo de reducir el disconfort térmico de los usuarios sin la necesidad del uso de sistemas activos. Otras medidas utilizan técnicas de diseño pasivo, las cuales son las encargadas de la reducción o la ganancia de calor a través de la cubierta (Torres, Viñachi, Cusquillo, Pazmiño y Segarra, 2019); entre las más usadas se encuentran: las pinturas de aislante térmico, los techos verdes (Garnica, 2020) y las máscaras por medio de protecciones solares como árboles, edificios, lamas, doble piel (Rojas, Soto y Díaz, 2020; Balter, Ganem y Discoli, 2016). Sin embargo, de todas estas gamas de estrategias abocadas a solucionar el confort de los espacios, la mayoría solo se enfoca en darle solución al confort térmico, dejando de lado los problemas lumínicos. Esta contrariedad se viene acrecentando desde la primera revolución industrial (1760-1840), como efecto de las transformaciones tecnológicas (Belén y López, 2016), al priorizarse la búsqueda de alternativas energéticas tradicionales, desde los navíos romanos que trasladaban leña, hasta la explotación de pozos petroleros. Ahora bien, como es consabido, los suministros de combustibles fósiles se acabarán mientras que el sol seguirá calentando a las generaciones futuras (Izzo, 2017). De ahí deriva la importancia del estudio de la captación de energía solar.

\section{ENERGÍA SOLAR: FORMA Y CAPTACIÓN}

El sol es el eje fundamental en la evolución de la vida en la tierra, ya que proporciona las condiciones adecuadas para la existencia de los seres vivos. Este astro se involucra en todos los ciclos productivos e, incluso, determina nuestras actividades progresistas en función del tiempo. Por esta razón, los seres humanos han tenido la necesidad de utilizar y aprovechar el sol para poder coexistir con el medio ambiente: como defensa ante el frío, para definir los tiempos de cosecha y hasta como promotor de vitamina D (Yépez, 2018). En la actualidad, no obstante, nuestras acciones ya no son regidas por el sol. Hoy, la luz artificial permite la iluminación permanente de espacios al interior de la vivienda, lo que ha causado alteraciones en el reloj biológico, los ciclos circadianos, las horas de alimentación, de trabajo y descanso; trastornos que son considerados también agentes de la epidemia de obesidad que afronta la sociedad (Iluminet, 2015).

Desde las primeras regulaciones urbanas en la antigua Grecia en la ciudad de Olynthus, se diseñó una trama urbano-lógica orientada de oriente a poniente, con el fin de organizar franjas y fachadas de viviendas mirando hacia al sur, para garantizar el acceso solar y mejorar la calidad del hábitat. Es importante destacar el Panteón de Adriano en Roma del año 126 d.C., ya que por su casi perfecta orientación cinco grados al N-NO, expone toda su forma arquitectónica vinculada íntimamente a la luz del sol, lo que provoca un juego de luces y sombras que para muchos historiadores marcó una nueva concepción espacial al interior (Linares, 2015).
En el presente, existen normativas y ordenanzas adaptadas a las necesidades de cada nación sobre el derecho al acceso solar o a la continua disponibilidad de luz solar directa sin obstrucciones (Contardo, Cecchi y Lara, 2017).

En una investigación a escala internacional en 10 países y 34 casos de estudio, sobre la influencia de la energía solar en la planificación urbana, se determinó que la selección de la tecnología y aceptación social en los tipos de materiales juegan un rol primordial al momento de diseñar los nuevos tejidos urbanísticos, donde radica la base del impacto ambiental, la economía local y la oferta del mercado. Se concluyó que las características funcionales formales presentaron un patrón de bordes rectos y rayas paralelas en la distribución de los módulos solares; configuración de factor de forma más idónea para la captación o protección de energía solar (Lobaccaro et al., 2019). Otro estudio enfocado en la construcción de una guía metodología para el diseño de edificios solares pasivos económicos y la reducción de la utilización de los sistemas de calefacción, ventilación y aire acondicionado (HVAC), analizó las condiciones normales de un edificio para luego realizar modificaciones en sus componentes constructivos (techo y envolvente), a fin de optimizar el aprovechamiento del microclima y de evaluar la temperatura máxima alcanzada por el edificio durante las 24 horas del día y los factores que influyen en la temperatura del ambiente interior (Marín, 2012). En una investigación destinada a comprobar el aprovechamiento de la energía solar en el Área Metropolitana de Mendoza, cuyas condiciones climatológicas son similares al objeto de estudio de este trabajo, se determinó la estrecha relación entre la morfología urbana y el acceso solar: el factor de forma y el factor de ocupación del suelo constituyen los agentes que más determinan la regulación urbana. De ello se infiere que, a mayor densidad urbana, menor disponibilidad de energía solar (Cárdenas y Vásquez, 2017).

Uno de los ejes centrales de la arquitectura bioclimática es el enfoque solar, que obliga a planificar en profundidad la apertura de los vanos, los cuales son los responsables del aporte de calor-luz (Monroy, 2006) y, en ciertas ocasiones, de la ventilación de la edificación; aspecto que, desde luego, aumenta la sensación de confort del usuario, sin la necesidad de recurrir al uso de sistemas de HVAC. En cuanto a la iluminación, para alcanzar estándares de confort se debe cumplir ciertos criterios de diseño mencionados en la Guía técnica, entre el que destaca el aprovechamiento de la luz natural en la iluminación de edificios IDAE (2005): "la elección del lugar, orientación, forma y dimensiones del edificio, aprovechando las ventajas de la aportación de luz cenital" (p. 36). Por tal razón es que diseñar edificios que contemplen el uso de la luz cenital como fuente vital de iluminación, genere magnos beneficios en los usuarios, desde la regulación de la producción de melatonina, pasando por el confort visual (Ramírez y Orozco, 2015), hasta importantes ahorros energéticos (Villalba, Pattini y Córica, 2012). Por consiguiente, surge la pregunta: ¿Si se diseñan prototipos de cubiertas ligeras, combinando 
el material zinc con el PVC, aumentará el nivel de iluminación y el confort térmico? Con el propósito de responder tal inquietud, se construyeron prototipos con materiales de la nueva era vernácula para zonas con climas cálidos, bajo los parámetros de sostenibilidad y protección al medio ambiente. Se busca que ellos funcionen como una base teórica para un diseño de techumbres capaz de permitir el paso de la luz solar con los menores aporte de calor, contribuyendo, de esta manera, a mejorar la habitabilidad y calidad de las viviendas de interés social.

\section{METODOLOGÍA}

El presente estudio fue llevado a cabo a través de un enfoque experimental, con un alcance descriptivo, y se inserta en la línea investigativa del confort al interior de la vivienda. Se efectuó en la región central de la Costa ecuatoriana, en la ciudad de Portoviejo, extendida en las coordenadas $\mathrm{S}^{\circ} 3^{\prime} 16.49^{\prime \prime}$ O80 $27^{\prime} 16.02^{\prime \prime}$ (Antipodas, 2020). La experimentación se realizó entre los meses de enero y marzo de 2021, cuando la temperatura máxima fue de $29^{\circ} \mathrm{C}$ y la mínima, de $23^{\circ} \mathrm{C}$; la nubosidad estuvo sobre el $75 \%$; las horas de luz fueron constantes, con un valor de 12 horas y 9 minutos; y la dirección de los vientos procedía del oeste (Tabla 1). El estudio fue dividido en 2 etapas.

\section{ETAPA 1 (ESTUDIO DE CAMPO)}

En la primera etapa metodológica, se utilizó la técnica de la observación estructurada, donde se desvelaron las características edilicias del universo geográfico (Cantón de Portoviejo) y se limitó el objeto de estudio a viviendas a un agua con cubiertas de Aluzinc. Se analizó una muestra de 308 soluciones habitacionales de la zona urbana y 89 viviendas de la zona rural. Este análisis evidenció que, en la zona urbana, el uso de material translúcido en las cubiertas (Mtc) representaba el 7,8\% de la muestra y, en la zona rural, el $5,6 \%$; lo que suma un total de 29 viviendas de las 397 analizadas.

El Cantón de Portoviejo está conformada por 9 parroquias urbanas y 7 parroquias rurales (Figura 2), donde se encuentran 70.428 viviendas, de las cuales 51.851 están ubicadas en la zona urbana y 18. 577, en la zona rural. De estas, 48.744 presentan como material de cubierta a la lámina de Aluzinc: 37.855 en la zona urbana y 10.889 en la zona rural (Tabla 2) (Instituto Nacional de Estadística y Censos [INEC], 2010).

\section{FÓRMULA PARA EL CÁLCULO DE LA MUESTRA}

Para el cálculo de la parte del universo que reunía las características de la población, se acudió a la fórmula probabilística planteada por Flores (2014) e indicada en la ecuación 1:

\begin{tabular}{|c|c|c|c|}
\hline $\begin{array}{c}\text { Agentes / } \\
\text { meses }\end{array}$ & Enero & Febrero & Marzo \\
\hline Temperatura & \multicolumn{2}{|c|}{ Máxima de $29^{\circ} \mathrm{C}$ y mínima de $23^{\circ} \mathrm{C}$} \\
\hline Nubosidad & $75 \%-81 \%$ nublado o mayormente nublado \\
\hline Precipitaciones & $24 \%-54 \%$ & $55 \%-60 \%$ & $60 \%-40 \%$ \\
\hline Horas de luz & \multicolumn{3}{|c|}{12 horas 9 min } \\
\hline Humedad & $93 \%-99 \%$ & $99 \%-100 \%$ & $100 \% \mathrm{Kte}$ \\
\hline $\begin{array}{c}\text { Viento } \\
\text { Hirección viento }\end{array}$ & $\begin{array}{c}14,2 \mathrm{a} 11,8 \\
\mathrm{~km} / \mathrm{h}\end{array}$ & $\begin{array}{c}11,7 \text { a } 10,3 \\
\mathrm{~km} / \mathrm{h}\end{array}$ & $\begin{array}{c}10,1 \mathrm{~km} / \mathrm{h} \\
\mathrm{Kte}\end{array}$ \\
\hline $\begin{array}{c}\text { Oeste } 76 \% \\
\text { Dadiación onda } \\
\text { corta }\end{array}$ & $5,6 \mathrm{kWh}$ luz visible y radiación ultravioleta. $76 \%$ & Oeste $75 \%$ \\
\hline
\end{tabular}

Tabla 1. Factores externos climatológicos. Fuente: Elaborado por los autores.

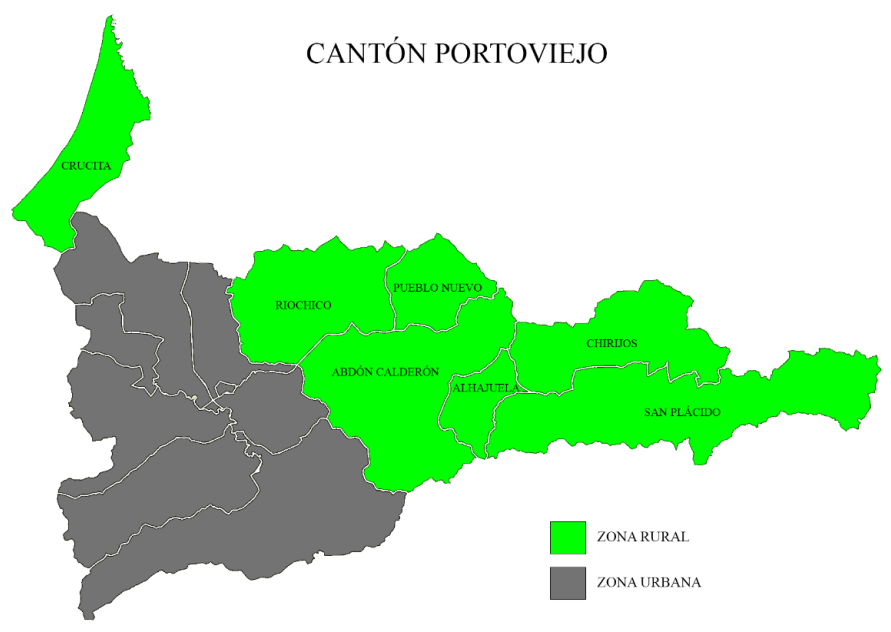

Figura 2. Mapa del Cantón Portoviejo. Fuente: Elaborado por los autores.

$$
n=\frac{P Q * N}{(N-1) \frac{E^{2}}{K^{2}}+P Q}
$$

Donde:

$\mathrm{n}$ : Es el tamaño de la muestra probabilística cuyo valor se busca.

$\mathrm{N}$ : Es el tamaño de la población total.

$\mathrm{PQ}$ : Es una constante de 0.25 de la varianza poblacional.

E: Es el error máximo admisible que varía de 0.01 a 0.09 .

$\mathrm{K}$ : Es el coeficiente de corrección del error es una constante igual a 2.

$(\mathrm{N}-1)$ : Es una constante relativa para muestras mayores a 30 (Flores, pp. 120-121). 


\section{CÁLCULO DEL TAMAÑO DE LA MUESTRA DE LAS PARROQUIAS.}

Para definir el número de parroquias a estudiar, se utilizó un error admisible del $1.16 \%$ de 16 parroquias, lo cual dio como resultado el $100 \%$ de estas, como se puede constatar en la ecuación 2.

$$
\mathrm{n}=\frac{P Q * \mathrm{~N}}{\frac{\mathrm{E}^{2}}{\mathrm{~K}^{2}}+\mathrm{PQ}}=\frac{0.25 * 16}{\frac{0.016^{2}}{2^{2}}+0.25}=\frac{4}{0.25}=16 .
$$

\section{CÁLCULO DEL TAMAÑO DE LA MUESTRA POR ZONAS}

\begin{tabular}{|c|c|c|}
\hline Zona & Orden & Cubierta de zinc (frecuencia) \\
\hline Urbana & 9 & 37.855 \\
\hline Rural & 7 & 10.889 \\
\hline Total & 16 & 48.744 \\
\hline
\end{tabular}

Tabla 2. Número de viviendas por zonas. Fuente. Elaborado por los autores.

Con el objetivo de calcular el número de soluciones habitacionales a evaluar, se empleó un error admisible del $5 \%$ de 48.744 viviendas, lo que dio como resultado 397 viviendas (véase ecuación 3); luego, se procedió a calcular la muestra por cuota para las diferentes zonas (véase Tabla 3) y se realizó una muestra estratificada para la zona rural (Tabla 4), dada la dispersión geográfica de la zona rural. Para finalizar esta primera etapa, se describieron las zonas, la frecuencia, el tamaño de la muestra y el Mtc (Tabla 5).

$$
\mathrm{n}=\frac{P Q * \mathrm{~N}}{(N-1) \frac{\mathrm{E}^{2}}{\mathrm{~K}^{2}}+\mathrm{PQ}}=\frac{0.25 * 48.744}{(48.744-1) \frac{0.05^{2}}{2^{2}}+0.25}=\frac{12.186}{30.71}=397
$$

\begin{tabular}{|c|c|c|c|c|}
\hline Zona & Orden & Frecuencia & $\%$ & $\mathbf{C m}$ \\
\hline Urbana & 9 & 37.855 & 77.66 & 308 \\
\hline Rural & 7 & 10.889 & 22.34 & 89 \\
\hline Total & 16 & 48.744 & 100 & 397 \\
\hline
\end{tabular}

Tabla 3. Número de viviendas a evaluar por zonas. Fuente: Elaborado por los autores.

\begin{tabular}{|c|c|c|c|c|c|}
\hline Zona rural & Orden & Frecuencia & $\%$ & $\mathbf{C m}$ & Mtc \\
\hline Abdón C. & 1 & 3.104 & 28.51 & 25 & 2 \\
\hline Alhajuela & 1 & 807 & 7.41 & 7 & 0 \\
\hline Chirijos & 1 & 433 & 3.98 & 4 & 1 \\
\hline Crucita & 1 & 1.812 & 16.64 & 15 & 0 \\
\hline Pueblo N. & 1 & 658 & 6.04 & 5 & 1 \\
\hline Riochico & 1 & 2.626 & 24.12 & 21 & 1 \\
\hline San Plácido & 1 & 1.449 & 13.31 & 12 & 0 \\
\hline Total & 7 & 10.889 & 100 & 89 & 5 \\
\hline
\end{tabular}

Tabla 4. Estratificación de la zona rural. Fuente: Elaborado por los autores.

\begin{tabular}{|c|c|c|c|c|c|}
\hline Zona & Orden & Frecuencia & $\%$ & $\mathbf{C m}$ & Mtc \\
\hline Urbana & 9 & 37.855 & 77.66 & 308 & 24 \\
\hline Rural & 7 & 10.889 & 22.34 & 89 & 5 \\
\hline Total & 16 & 48.744 & 100 & 397 & 29 \\
\hline
\end{tabular}

Tabla 5. Número de viviendas que utilizan el material translúcido en las diferentes zonas del Cantón de Portoviejo. Fuente: Elaborado por los autores.

\section{ETAPA 2 (DISEÑO DE TESTIGOS Y MODELOS DE CUBIERTAS)}

Para evaluar las condiciones térmicas y lumínicas a través del estudio experimental, se construyeron 4 prototipos a escala con dimensiones $1 \mathrm{~m} * 1 \mathrm{~m} * 1 \mathrm{~m}$, de paredes de plywood. Las variables analizadas fueron las características de iluminación y la temperatura al interior de los prototipos. El primer modelo (A) corresponde a un modelo base, con una cubierta $100 \%$ metálica, y sirvió para evaluar las condiciones ambientales y compararlas con los otros tres prototipos. El modelo (B) fue diseñado a base de las soluciones empíricas de iluminación cenital de las 29 viviendas observadas, las cuales optaron por incorporar el material translucido a base de PVC en la parte superior central de sus moradas, en el que el PVC representó el $2 \%$ del elemento cubierta. El modelo (C) fue elaborado en PVC y representó el 10\% del total de la cubierta (dos franjas centrales de 5\%; una horizontal y la otra perpendicular a la trayectoria solar), formando una cruz en el centro de la cubierta. El modelo (D) fue diseñado a base de las recomendaciones de la guía técnica IDAE y los métodos de iluminación cenital de las 29 viviendas observadas, las cuales optaron por incorporar el material translucido a base de PVC en la parte lateral exterior de sus viviendas (garajes, pérgolas), en el que el PVC constituyó el $16 \%$ de la cubierta, representados en dos franjas laterales de $8 \%$, dispuestas horizontalmente respecto a la trayectoria solar. Los prototipos fueron orientados en el eje Este - Oeste con la parte más alta de la techumbre hacia el Oeste, con una pendiente de $10 \%$, y se posicionaron equidistantemente en el eje Norte - Sur, con una separación entre ellos de $1 \mathrm{~m}$. Los parámetros medidos en ellos fueron la temperatura del aire interior (Tai) y el nivel de iluminación interior (E), para lo cual se emplearon termohigrómetros digitales, HTC-2 y dos apps digitales; lux light meter doggo apps y luxómetro: smart lux meter.

\section{RESULTADOS Y DISCUSIÓN}

\section{ANÁLISIS DEL PROTOTIPO A Y B}

La toma de datos del prototipo $\mathrm{A}$ y $\mathrm{B}$ se registró durante 15 días del mes de enero del 2021, en 3 periodos diferentes $(1-7,10-14,29-31)$, de 08:00 h a 18:00 h, en intervalos de 60 minutos. En la Figura 3 se muestran los 


\begin{tabular}{|c|c|c|c|c|}
\hline \multirow{2}{*}{ horas } & \multicolumn{2}{|c|}{ Tai $/{ }^{\circ} \mathbf{C}$} & \multicolumn{2}{|c|}{$\mathbf{E} / \mathbf{L u x}$} \\
\hline & $\mathrm{A}$ & $\mathrm{B}$ & $\mathrm{A}$ & $\mathrm{B}$ \\
\hline $8: 00$ & 25,76 & 25,92 & 0 & 45,0 \\
\hline $9: 00$ & 26,07 & 26,14 & 0 & 45,9 \\
\hline $10: 00$ & 27,38 & 27,72 & 0 & 53,6 \\
\hline $11: 00$ & 29,85 & 30,05 & 0 & 53,9 \\
\hline $12: 00$ & 30,78 & 30,98 & 0 & 54,1 \\
\hline $13: 00$ & 32,23 & 33,93 & 0 & 54,5 \\
\hline $14: 00$ & 32,45 & 34,16 & 0 & 54,5 \\
\hline $15: 00$ & 32,27 & 33,97 & 0 & 54,5 \\
\hline $16: 00$ & 32,09 & 33,86 & 0 & 54,1 \\
\hline $17: 00$ & 30,15 & 30,35 & 0 & 50,7 \\
\hline $18: 00$ & 29,19 & 29,38 & 0 & 42,3 \\
\hline
\end{tabular}

Figura 3. Matriz comparativa entre el prototipo A y B. Fuente: Elaborado por los autores.

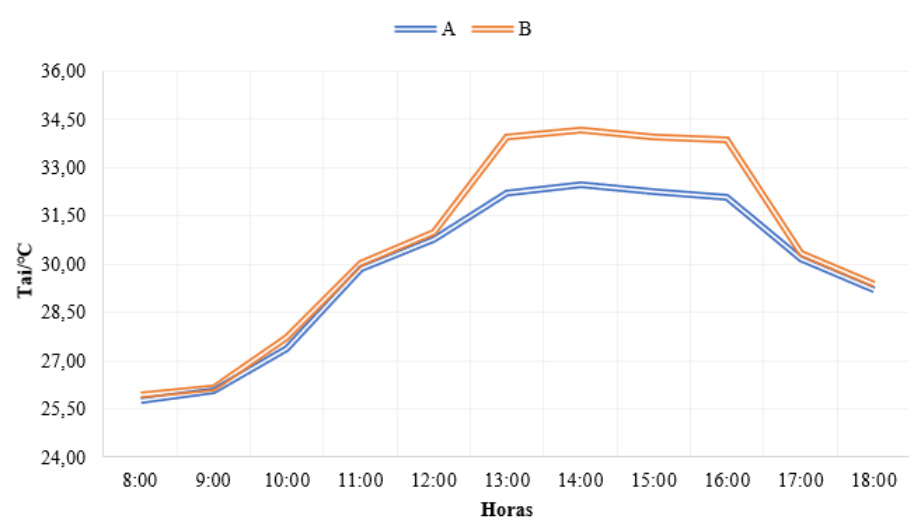

Figura 4. Curvas comparativas de la temperatura interior entre el modelo base A y el prototipo B. Fuente: Elaborado por los autores.

$\square \mathrm{B}$

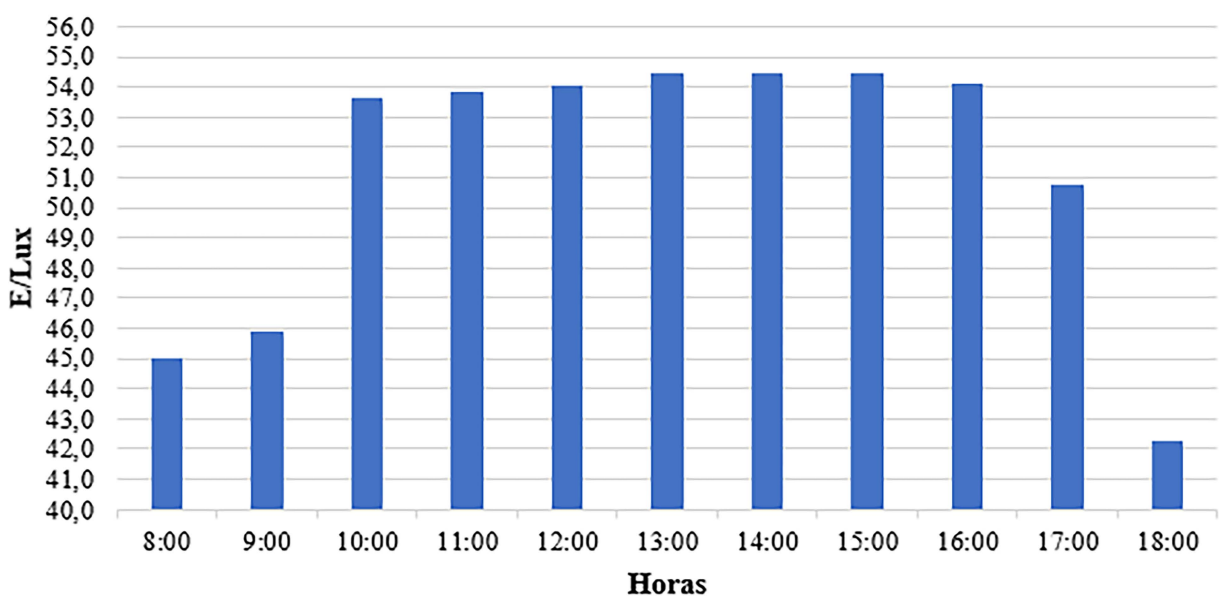

Figura 5. Niveles de iluminación interior del modelo base A y el prototipo B. Fuente: Elaborado por los autores.

resultados promedio de todas las mediciones tomadas en cada una de las horas de medición y la estructura del prototipo A y B.

De acuerdo a los resultados obtenidos del modelo base $A$ y el prototipo $B$, la diferencia promedio diaria de temperatura interior entre ellos es de $0,75^{\circ} \mathrm{C}$. La temperatura máxima del modelo $\mathrm{B}$ es de $34,16^{\circ} \mathrm{C}$, registrada alrededor de las $14: 00 \mathrm{~h}$, la cual es $1,71^{\circ} \mathrm{C}$ mayor que la temperatura interior del modelo (A). La máxima diferencia de temperatura interna a las $16: 00 \mathrm{~h}$ fue de un valor de $1,77^{\circ} \mathrm{C}$ (Figura 4). Por otra parte, se determinó que el valor máximo de luxes del prototipo $B$ fue de 54,5 luxes, desde las 13 pm hasta las 3 pm aproximadamente; $y$, en cuanto al modelo (A), el modelo base, este presentó un valor de 0 luxes (Figura 5).

\section{ANÁLISIS DEL PROTOTIPO A Y C}

La toma de datos del prototipo $\mathrm{A}$ y $\mathrm{C}$ se efectuó durante 15 días del mes de febrero del 2021, durante 3 periodos (5-14, 19-21, 26-28), de 08:00 h a 18:00 h, en intervalos de 60 minutos. En la Figura 6 se ilustran los resultados promedio de todas las mediciones tomadas en cada una de las horas de medición y la estructura del prototipo $A$ y C.

Conforme a los resultados obtenidos en el modelo base (A) y el prototipo $C$, la diferencia promedio diaria de temperatura interior entre ellos es de $1,25^{\circ} \mathrm{C}$. La temperatura máxima del modelo $\mathrm{C}$ es de $36,57^{\circ} \mathrm{C}$ alrededor de las $14: 00 \mathrm{~h}$, la cual es $4,53^{\circ} \mathrm{C}$ mayor que la temperatura interior del modelo (A) (Figura 7). Asimismo, se determinó que el valor máximo de luxes del prototipo 


\begin{tabular}{|c|c|c|c|c|}
\hline \multirow{2}{*}{ horas } & \multicolumn{2}{|c|}{ Tai/ ${ }^{\circ} \mathbf{C}$} & \multicolumn{1}{|c|}{ E/Lux } \\
\cline { 2 - 4 } & $\mathrm{A}$ & $\mathrm{C}$ & $\mathrm{A}$ & $\mathrm{C}$ \\
\hline $8: 00$ & 25,72 & 25,88 & 0 & 64,6 \\
\hline $9: 00$ & 26,16 & 26,32 & 0 & 67,6 \\
\hline $10: 00$ & 27,47 & 28,39 & 0 & 69,9 \\
\hline $11: 00$ & 29,95 & 30,15 & 0 & 70,5 \\
\hline $12: 00$ & 30,85 & 31,13 & 0 & 70,5 \\
\hline $13: 00$ & 31,96 & 34,35 & 0 & 71,3 \\
\hline $14: 00$ & 32,04 & 36,57 & 0 & 71,2 \\
\hline $15: 00$ & 31,77 & 34,18 & 0 & 70,8 \\
\hline $16: 00$ & 31,74 & 33,47 & 0 & 69,7 \\
\hline $17: 00$ & 29,91 & 30,26 & 0 & 69,5 \\
\hline $18: 00$ & 28,69 & 29,26 & 0 & 57,9 \\
\hline
\end{tabular}

Figura 6. Matriz comparativa entre el prototipo A y C. Fuente: Elaborado por los autores.

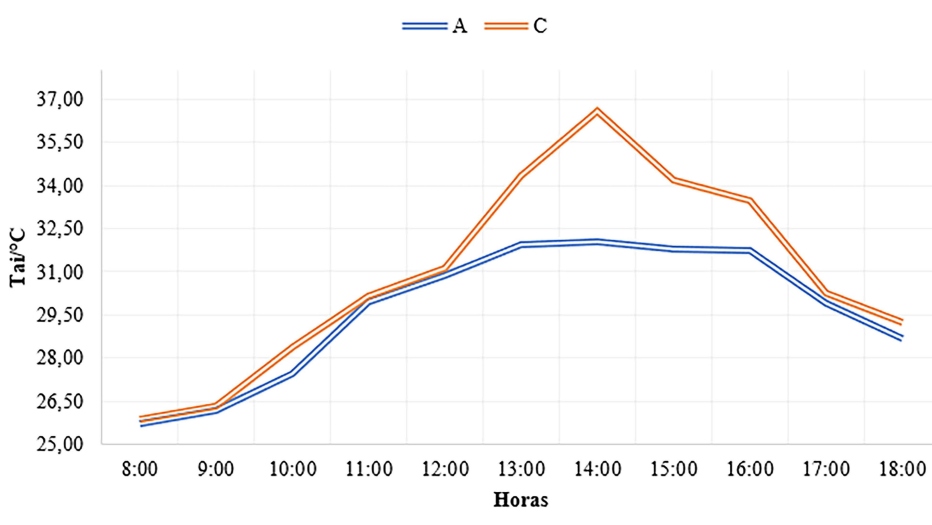

Figura 7. Curvas comparativas de la temperatura interior entre el modelo base A y el prototipo B. Fuente: Elaborado por los autores.

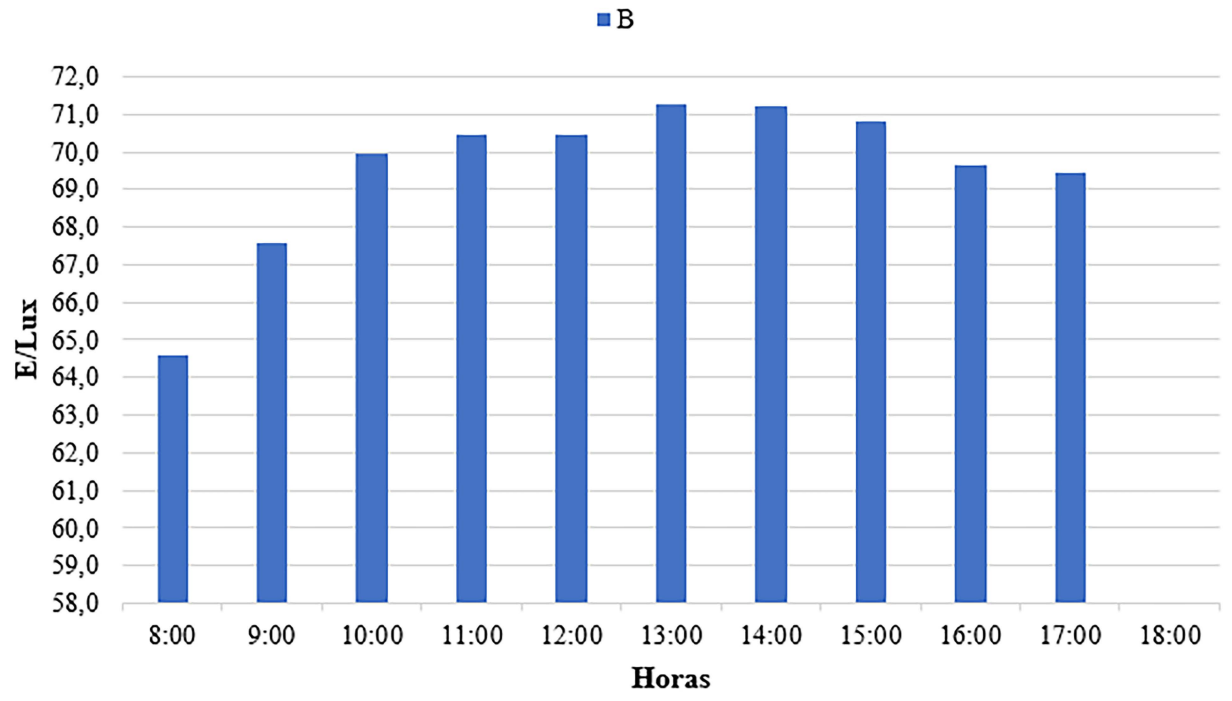

Figura 8. Niveles de iluminación interior del modelo base A y el prototipo C. Fuente: Elaborado por los autores.

C fue de 71,3 luxes, a las 13:00 h aproximadamente; $y$, en cuanto al modelo (A), el modelo base, este presentó un valor de 0 luxes (Figura 8).

\section{CÁLCULO DE LAS VARIACIONES DEL PROTOTIPO A Y D}

La toma de datos del prototipo A y $D$ se llevó a cabo durante 15 días del mes de marzo de 2021, en 2 periodos diferentes $(6-12,19-26)$, desde las 08:00 h a las 18:00 h, en intervalos de 60 minutos. En la Figura 9 se observan los resultados promedio de todas las mediciones tomadas en cada una de las horas de medición y la estructura del prototipo A y D.

Según los resultados obtenidos a partir de la comparación entre el modelo base (A) y el prototipo $D$, la diferencia promedio diaria de temperatura interior entre ellos es de $1,90^{\circ} \mathrm{C}$. La temperatura máxima del modelo $\mathrm{D}$ es de $38,26^{\circ} \mathrm{C}$, registrada alrededor de las $13: 00 \mathrm{~h}$, la cual es $5,07^{\circ} \mathrm{C}$ mayor que la temperatura interior del modelo (A) (Figura 10). Se determinó, además, que el valor máximo de luxes del prototipo $D$ fue de 118,4 luxes, a las 13:00 $\mathrm{h}$ aproximadamente y que el modelo (A), el modelo base, presentó un valor de 0 luxes (Figura 11).

\section{COMPARACIÓN DE LAS ALTERNATIVAS DE DISEÑO}

Bajo este contexto de análisis, se logró determinar que, desde las 13:00 h hasta las 14:00 h, aproximadamente, se da la mayor influencia de radiación solar sobre el elemento cubierta; resultado que concuerda con el de Salgado (2012), cuya investigación determinó que, en condiciones normales, la interfase desde el mediodía necesaria para 


\begin{tabular}{c|c|c|c|c|}
\hline \multirow{2}{*}{ horas } & \multicolumn{2}{|c|}{ Tai/ ${ }^{\circ} \mathbf{C}$} & \multicolumn{2}{|c|}{ E/Lux } \\
\hline & $\mathrm{A}$ & $\mathrm{D}$ & $\mathrm{A}$ & $\mathrm{D}$ \\
\hline $8: 00$ & 25,89 & 26,05 & 0 & 87,4 \\
\hline $9: 00$ & 26,43 & 26,59 & 0 & 90,3 \\
\hline $10: 00$ & 28,47 & 30,66 & 0 & 101,7 \\
\hline $11: 00$ & 30,46 & 32,25 & 0 & 103,3 \\
\hline $12: 00$ & 31,89 & 35,85 & 0 & 109,3 \\
\hline $13: 00$ & 33,19 & 38,26 & 0 & 118,4 \\
\hline $14: 00$ & 34,47 & 37,73 & 0 & 114,3 \\
\hline $15: 00$ & 32,17 & 34,45 & 0 & 114,2 \\
\hline $16: 00$ & 31,71 & 33,47 & 0 & 109,7 \\
\hline $17: 00$ & 30,01 & 30,27 & 0 & 104,1 \\
\hline $18: 00$ & 28,75 & 29,12 & 0 & 83,2 \\
\hline
\end{tabular}

Figura 9: Matriz comparativa entre la chapa A y D. Fuente: Elaborado por los autores.

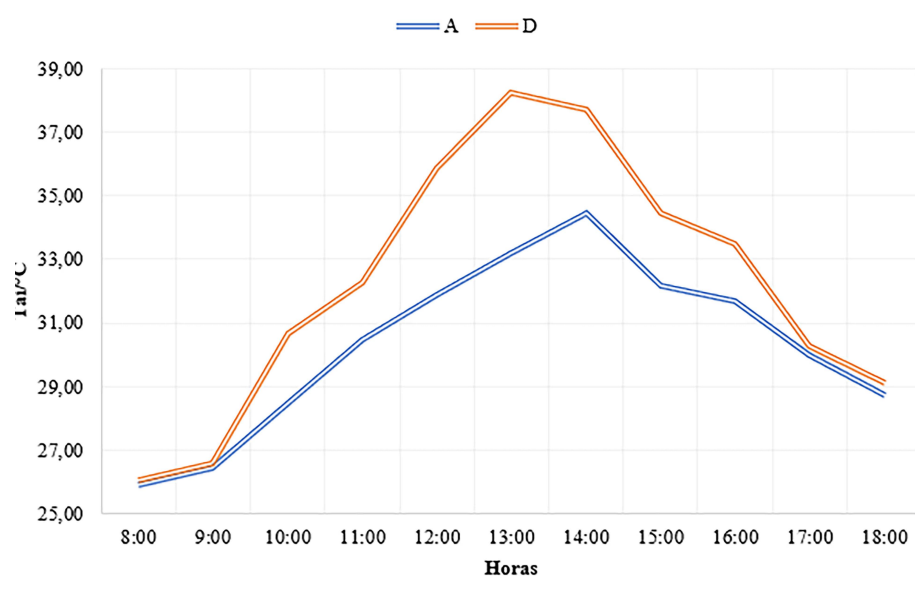

Figura 10. Curvas comparativas de la temperatura interior entre el modelo base A y el prototipo D. Fuente: Elaborado por los autores.

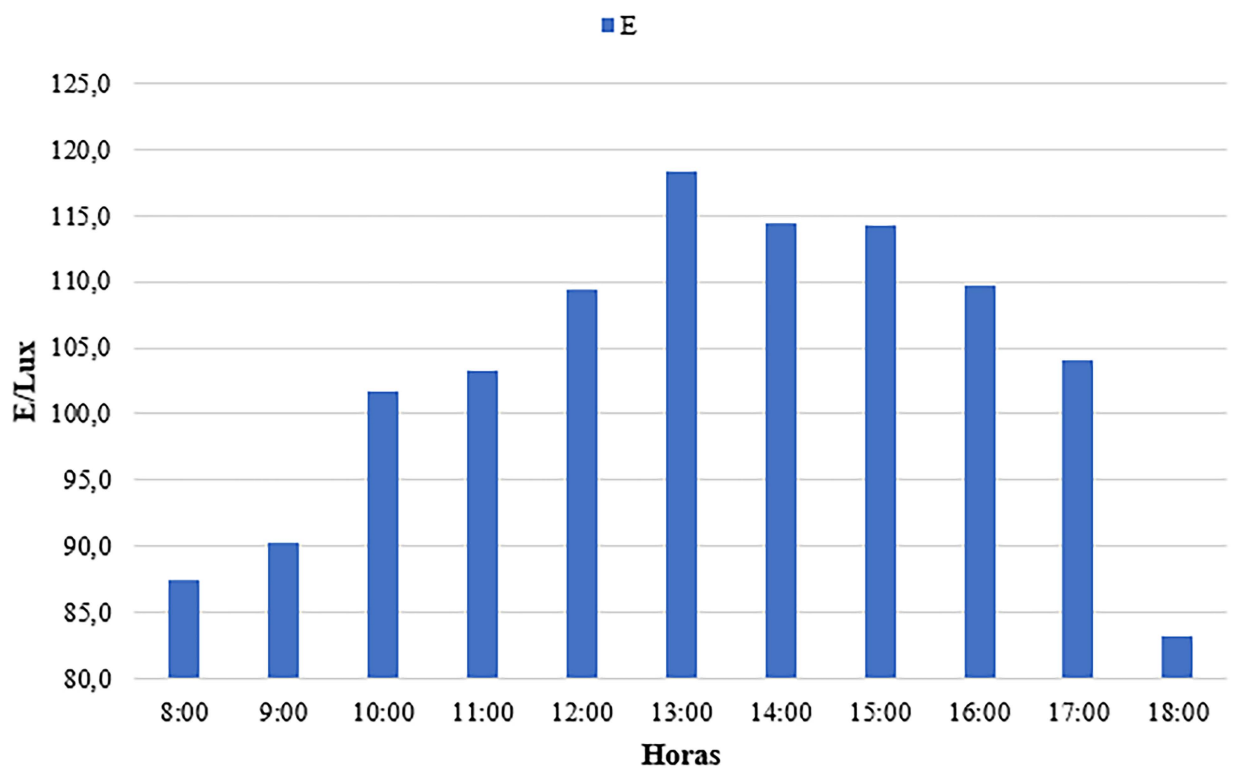

Figura 11. Niveles de iluminación interior del modelo base A y el prototipo D. Fuente: Elaborado por los autores.

que las edificaciones alcanzaran su máxima temperatura era de 1 hora, 34 minutos y 10 segundos. En base al presente estudio se destaca lo siguiente: el modelo B presenta la menor variación interna de temperatura, con un $32,63 \%$, que contrastan con los $32,97 \%$ y los $34,40 \%$ del modelo C y D, respectivamente. Sin embargo, desde el momento en donde los modelos alcanzan la máxima temperatura, el modelo D, presenta descensos drásticos de temperatura alcanzando un relativo equilibrio a las 15:30 h aproximadamente (Figura 12), en relación con los otros prototipos. Además, este modelo presenta valores que sobrepasan los 100 lux desde las 10:00 h a las 17:00 h (Figura 13). Este fenómeno se debe a la cantidad de Mtc, $(16 \%)$ con la que fue diseñado; porcentaje que supera el $10 \%$ recomendado por $\operatorname{IDAE}(2005$, p. 48$)$ y criterio corroborado por Monroy (2006) quien sostiene que los criterios de diseño planteados para ventanas verticales también se pueden adaptar para el diseño de huecos de cubierta (p. 80), especificando que la relación $1 / 10$ es satisfactoria.

En términos cualitativos, el modelo C, diseñado estrictamente con la configuración de factor de forma a la zona ocupada del $10 \%$ y con una forma de cruz, no presenta una buena distribución de la luz interior. Lo contrario sucede con el modelo $D$ que sí proyecta una adecuada distribución de luz al interior, al presentar dos franjas laterales y la peculiaridad de que la parte más angosta forma una perpendicular con la trayectoria solar; aspecto medible y observable que asemeja la distribución espacial de las ciudades de la antigua Grecia, famosas por una orientación que garantizaban el acceso continuo de luz solar. Esta configuración concuerda, igualmente, con Lobaccaro et al. (2019), quienes analizaron 34 casos de 


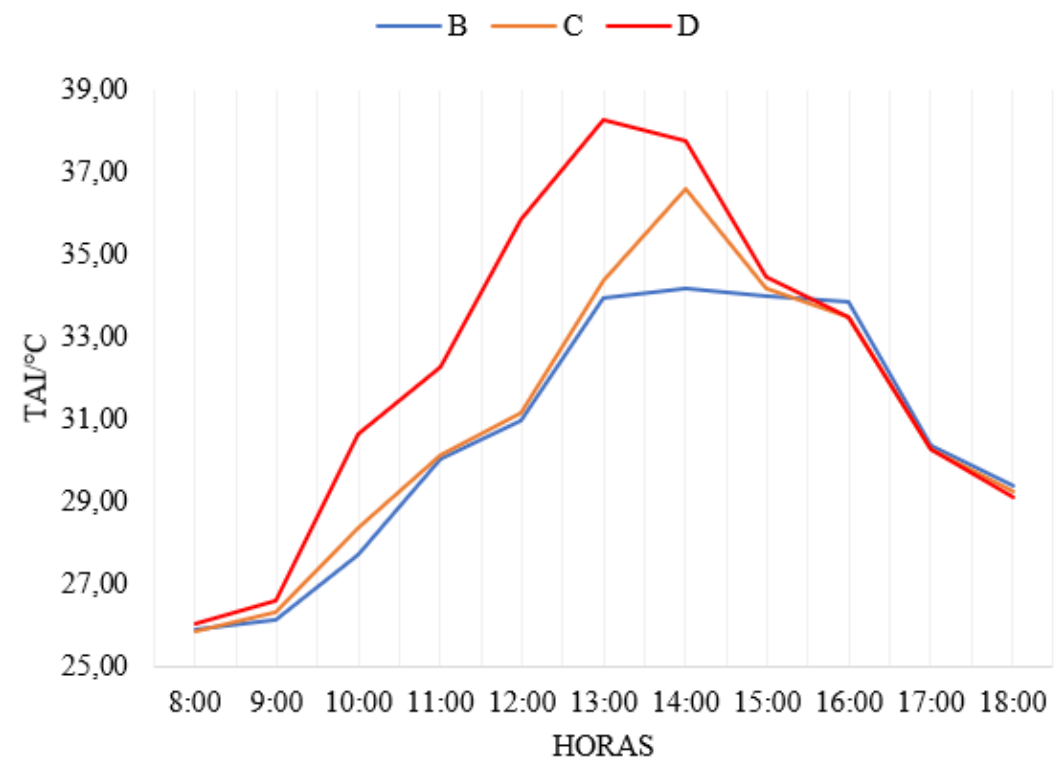

Figura 12. Temperatura al interior de los modelos B, C y D. Fuente: Elaborado por los autores.

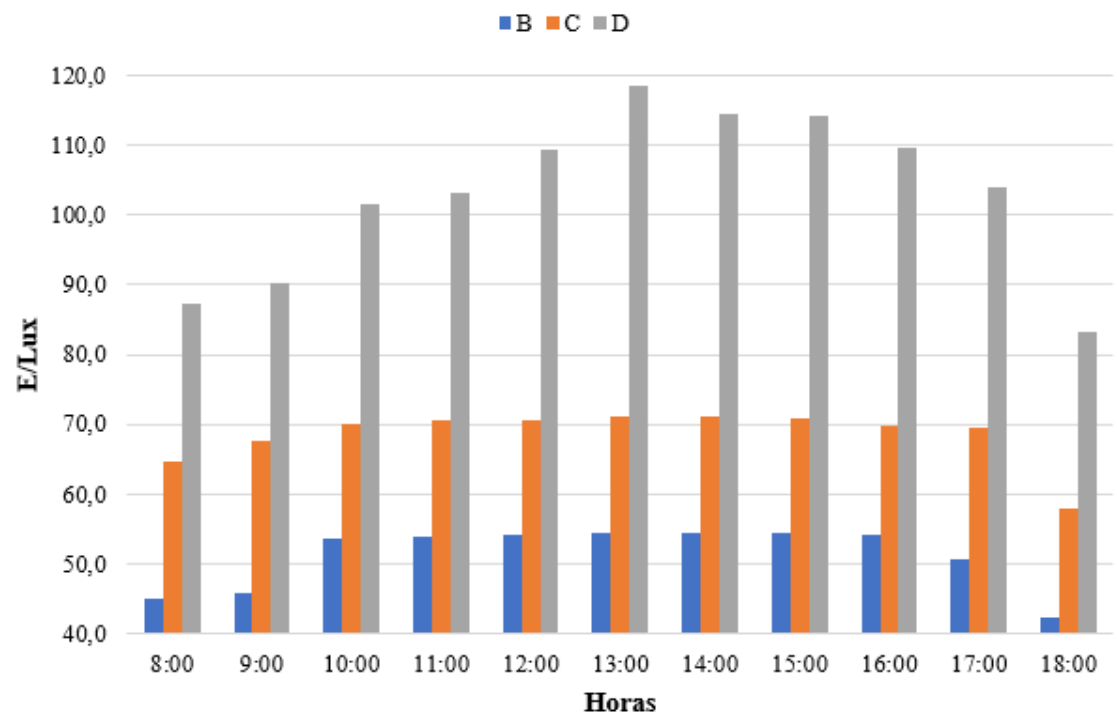

Figura 13. Niveles de iluminación al interior de los modelos B, C y D. Fuente: Elaborado por los autores.

estudios en 10 países diferentes, determinando que, en términos funcionales de factor de forma, la distribución en bordes rectos para la captación de energía solar brinda mayores resultados. En suma, a partir de los criterios mencionados y la experimentación realizada, esta configuración exhibe la mejor distribución de la luz al interior, aunque para algunos autores, como Linares (2015) el juego entre luces y sombras producido por otras opciones seguirá siendo un paradigma.

\section{CONSIDERACIÓNES GENERALES}

Al ser un país en vías de desarrollo, Ecuador no cuenta con una normativa u ordenanzas cantonales sobre el derecho al acceso solar, lo que se puede aprecia en su tejido urbanístico y, particularmente, en las 397 soluciones habitaciones observadas en esta investigación, de las cuales, solo el $13,4 \%$ utilizaban el material translúcido en las cubiertas para la captación de luz cenital. Cualitativamente, en la primera etapa del estudio, como parte de la observación sistemática, en las zonas rurales, se advirtieron viviendas extremadamente calientes y con ausencia de luz, las cuales estaban adosadas en sus retiros laterales y posteriores, lo que sumado al hacinamiento denotaba un bajo índice de habitabilidad. En referencia a las necesidades de la zona, se puede implementar la utilización del prototipo D. Asimismo, se pudo corroborar que el 
material de cubierta predominante en el Cantón es el Aluzinc, que incluso es utilizado como doble cubierta.

Con respecto a la aplicabilidad de los prototipos, el modelo $\mathrm{B}$ se recomienda para ganancias de luz cenital puntuales. El modelo $C$ no se recomienda y el modelo $D$, para viviendas que cuenten con algún tipo de protección solar y para viviendas adosadas en retiros laterales y posteriores.

\section{CONCLUSIÓN}

En este trabajo se evaluaron tres alternativas de prototipos de cubiertas ligeras, con la combinación de los materiales Zincy PVC, para zonas con climas cálidos bajo los parámetros de sostenibilidad y protección al medio ambiente. Para ello, se utilizaron estrategias pasivas de captación de iluminación cenital, tales como la orientación y la compacidad de factor de forma, implementando la estrategia del enfoque solar, lo que permite diseñar cubiertas ligeras que mejoraren el aporte de luz al interior de la vivienda sin generar espacios con sobrecalentamientos. Este proceso metodológico que se basó en mediciones de campo a partir de modelos a escala tuvo como descubrimiento más importante la determinación de la hora de mayor influencia de sol sobre las cubiertas para este tipo de zonas y la configuración que se le debe dar al Mtc, para diseñar espacios que cumplan con adecuadas características térmicas y lumínicas en pos de viviendas saludables. Una de las limitantes del proceso investigativo fue la toma de datos de luxes, por su alto índice de variabilidad en el espacio tiempo.

Lo anterior permite desechar en cierto grado la hipótesis planteada, ya que la incorporación de Mtc en la cubierta, aumenta el nivel de iluminación, pero su configuración de factor de forma no disminuye ni tampoco mantiene la temperatura al interior de los modelos ensayados. Ahora bien, los resultados del modelo $\mathrm{D}$ presentan descensos drásticos de temperatura desde el momento en que alcanza su clímax y, a su vez, registran la mejor distribución de luz al interior, es decir, el mejor el nivel de luxes. A modo de conclusión, es importante mencionar que surge la interrogante acerca de qué sucedería si se aplican estas estrategias constructivas en cubiertas de doble piel o en cubiertas a dos aguas. Por consiguiente, como siguiente paso de perfeccionamiento de los prototipos aquí expuestos, este estudio recomienda el análisis de la incorporación del material translúcido en cubiertas a dos aguas.

\section{AGRADECIMIENTOS}

Los autores agradecen a la Universidad Técnica de Manabí y al Instituto de Posgrado-UTM - Se agradece la beca de investigación dentro de la Maestría en Ingeniería Civil Mención Construcción de Vivienda Social. Resolución: RPC-SO-10-No.130-2019 - Se agradece a la Universidad Católica de Cuenca, proyecto DAMA-215543.

\section{REFERENCIAS BIBLIOGRÁFICAS}

Alchapar, N., Correa, E. y Cantón, M. (2012). Índice de reflectancia solar de revestimientos verticales: Potencial para la mitigación de la isla de calor urbana. Ambiente Construido, 12(3), 107-123. DOI: https://doi.org/10.1590/S167886212012000300008

Antipodas (2020). Coordenadas de Portoviejo (Ecuador). Recuperado de https://www.antipodas.net/coordenadaspais/ ecuador/portoviejo.php

Balter, J., Ganem, C. y Discoli, C. (2016). Ahorros en los consumos para enfriamiento de espacios interiores mediante la evaluación de sistemas de protección solar en edificios residenciales en altura. El caso de la "ciudad-oasis" de Mendoza, Argentina. Hábitat Sustentable, 6(1), 72-83. Recuperado de http://revistas.ubiobio.cl/index.php/RHS/article/view/2353

Belén, B. y López, J. (2016). Revolución industrial. Recuperado de https://economipedia.com/definiciones/primerarevolucion-industrial.html

Cárdenas, L. Y Vásquez, P. (2017). Potencial solar en fachadas integrando la densidad urbana: Una mirada crítica a la norma urbanística chilena. AUS [Arquitectura / Urbanismo / Sustentabilidad], 18, 58-63. DOI: https://doi.org/10.4206/ aus.2015.n18-10

Contardo, J., Cecchi, C. y Lara, K. (2017). Acceso Solar: Un derecho urbano para la calidad de vida vulnerado desde la Gentrificación contemporánea. El caso de la comuna de Estación Central, Chile. Revista 180, 0(39). DOI: http://dx.doi. org/10.32995/rev180.Num-39.(2017).art-283

Couret, D. y Párraga, J. (2019). Evolución de la vivienda de interés social en Portoviejo. Cuadernos de Vivienda y Urbanismo, 12(23). DOI: https://doi.org/10.11144/Javeriana. cvu12-23.evis

El Diario (2018). Temperatura más alta en Portoviejo. El Diario. EDIASA. Recuperado de https://www.eldiario.ec/noticiasmanabi-ecuador/467707-temperatura-mas-alta-en-portoviejo/

El Universo (2020). Se registran altas temperaturas en Guayas y Manabí, este es el pronóstico para el viernes 30 de octubre. El Universo. Recuperado de https://www.eluniverso.com/ noticias/2020/10/29/nota/8031442/inamhi-pronosticotiempo-ecuador-30-octubre-ecuador-radiacion

Flores, C. (2014). Introducción a la Estadística. Manta: Mar Abierto.

Garnica, A. (2020). Efecto en la temperatura para mitigar la Isla de Calor utilizando dos ecotecnias. Meio Ambiente (Brasil), 2(5). Recuperado de http://www.meioambientebrasil.com.br/ index.php/MABRA/article/view/92

Hernández-Salomón, M., Rizo-Aguilera, L. y FrómetaSalas, Z. (2017). Recomendaciones de diseño con enfoque medioambiental para cubiertas ligeras en edificaciones del centro histórico de Santiago de Cuba. Ciencia en su PC, (1), 70-88. Recuperado de https://www.redalyc.org/articulo. oa?id $=181351125006$ 
IDAE (2005). Guía técnica: Aprovechamiento de la luz natural en la iluminación de edificios. Recuperado de https://www.idae.es/ publicaciones/guia-tecnica-aprovechamiento-de-la-luz-naturalen-la-iluminacion-de-edificios

lluminet (2015). El equilibrio entre luz artificial y natural puede ser una clave contra la obesidad. Iluminet. Revista de iluminación. Recuperado de https://www.iluminet.com/iluminacion-artificialobesidad/

Instituto Nacional de Estadística y Censos [INEC] (2010). Población y Demografía. Recuperado de https://www.ecuadorencifras.gob. ec/censo-de-poblacion-y-vivienda/

Instituto Nacional de Meteorología e Hidrología [INAMHI] (2014). Análisis Climatológicos. Recuperado de http://www. serviciometeorologico.gob.ec/meteorologia/bolhist/cli/2014/ MENSUAL/bol_jul_2014.pdf

Izzo, G. (2017). Perspectiva de la energía nuclear en la futura matriz energética. Tesis de magíster. Universidad Nacional de La Plata. DOI: https://doi.org/10.35537/10915/61231

Linares, T. (2015). Further observations on the light in the Pantheon in Rome. VLC arquitectura. Research Journal, 2(1), 33-55. DOI: https://doi.org/10.4995/vlc.2015.3376

Lobaccaro, G., Croce, S., Lindkvist, C., Probst, M., Scognamiglio, A., Dahlberg, J., Lundgren, M. y Wall, M. (2019). A cross-country perspective on solar energy in urban planning: Lessons learned from international case studies. Renewable and Sustainable Energy Reviews, 108, 209-237. DOI: https://doi.org/10.1016/j. rser.2019.03.041

Macías, A. (2018). Análisis de los Factores Endógenos y Exógenos Higrotérmicos del Sector los Bosques Cantón Portoviejo y Propuesta de Solución. Tesis de doctorado. Universidad laica "Eloy Alfaro" de Manabí. Recuperado de https://repositorio. uleam.edu.ec/handle/123456789/2330

Marín, F. (2012). Evaluación del rendimiento de calefacción o refrescamiento producido por los elementos constructivos y microclima de una vivienda pasiva. Una forma de integrar el rendimiento del confort térmico pasivo a su administración del ciclo de vida de un edificio. Revista INVI, 27(75), 171-197. Recuperado de https://revistainvi.uchile.cl/index.php/INVI/ article/view/62339/66418

Monroy, M. (2006). Manuales de Diseño ICARO. [Manual-1ILUMINACION]. Recuperado de https://m2db.wordpress. com/2014/09/01/manuales-de-diseno-icaro/

Oster, L. (2021). Astronomíamoderna. Recuperadodehttps://books. google.es/books?id=xpAXEAAAQBAJ\&hl=es\&source=gbs_ navlinks_s

Osuna-Motta, I., Herrera-Cáceres, C. y López-Bernal, O. (2017). Techo plantado como dispositivo de climatización pasiva en el trópico. Revista de Arquitectura (Bogotá), 19(1), 42-55. DOI: https://doi.org/10.14718/RevArq.2017.19.1.1109

Plasticseurope (2021). Tipos de plásticos. Recuperado de https:// www. plasticseurope.org/es/about-plastics/what-are-plastics/ large-family

Ramírez, A. y Orozco, A. (2015). La envolvente arquitectónica y su influencia en la iluminación natural. Hábitat Sustentable, 5(1), 44-53. Recuperado de http://revistas.ubiobio.cl/index.php/RHS/ article/view/1811

Rodríguez, S. (2017). Diseño y evaluación de un sistema de doble cubierta ventilada para reducir la transmisión de calor al interior de la vivienda media en Puerto Vallarta, Jalisco. Tesis de maestría. Universidad Autónoma Metropolitana, México. Recuperado de http://zaloamati.azc.uam.mx/handle/11191/5944

Rojas, J., Soto, P. y Díaz, H. (2020). Evaluación dinámica de control solar lumínico. Propuestas en una biblioteca de arquitectura moderna en Santiago. Estoa. Revista de la Facultad de Arquitectura y Urbanismo de la Universidad de Cuenca, 9(18), 125-137. DOI: https://doi.org/10.18537/est.v009.n018.a11

Sisternes, Á. (2019). Diseño pasivo: Concepto, ventajas y desventajas. Kömmerling. Recuperado de https://retokommerling. com/diseno-pasivo/

Torres, B., Viñachi, J., Cusquillo, J., Pazmiño, C. y Segarra, M. (2019). Aprovechamiento de la arquitectura pasiva para la reducción de carga térmica por ganancia solar en un edificio de la Facultad de Arquitectura de la Universidad de Guayaquil. Extensionismo, Innovación y Transferencia Tecnológica, 5(0), 378 388. DOI: https://doi.org/10.30972/eitt.503775

Torres, J., Coch, H. y Isalgué, A. (2019). Assessment of the reflectivity and emissivity impact on light metal roofs thermal behaviour, in warm and humid climate. Energy and Buildings, 188-189, 200208. DOI: https://doi.org/10.1016/j.enbuild.2019.02.022

Véliz-Párraga, J. y González-Couret, D. (2019). Vivienda de interés social en Portoviejo. ambiente térmico interior. AUS [Arquitectura / Urbanismo / Sustentabilidad], (26), 35-41. DOI: https://doi. org/10.4206/aus.2019.n26-07

Villalba, A., Pattini, A. y Córica, M. (2012). Análisis de las características morfológicas de las envolventes edilicias y del entorno urbano desde la perspectiva de la iluminación natural. Ambiente Construido, 12(4), 159-175. DOI: https://doi. org/10.1590/S1678-86212012000400011

Yépez, K. (2018). 10 beneficios de tomar el sol para nuestra salud. Elsevier Connect. Recuperado de https://www.elsevier.com/eses/connect/ciencia/beneficios-salud-del-sol 